\title{
Post-stroke rehabilitation
}

\author{
S Whitehead, $\mathrm{MB} \mathrm{ChB}$; E Baalbergen, $\mathrm{MB} \mathrm{ChB}$ \\ Life Rehabilitation Unit, Vincent Pallotti Hospital, Cape Town, South Africa
}

Corresponding author: S Whitehead (se_whitehead@yahoo.com)

This article offers insight into post-stroke rehabilitation. Stroke is a major public health issue with high mortality and morbidity rates, both nationally and internationally. Recent medical advances resulted in improvements in mortality rates, but the disabling longterm effects of stroke are still prevalent. Post-stroke rehabilitation improves patient outcomes by decreasing the chance of developing secondary complications and maximising the patient's independence despite their impairments. Post-stroke rehabilitation is delivered by an interdisciplinary team experienced in the rehabilitation process. There are specific focus areas during this process, which ensure a holistic approach. Early discharge planning is essential and can help to ease the transition from inpatient rehabilitation to a patient's home environment. Stroke recovery is heterogeneous and multilayered; therefore, it is difficult to accurately predict post-stroke outcomes, but the importance of early interventions and ongoing outpatient rehabilitation is stressed.

S Afr Med J 2019;109(2):81-83. DOI:10.7196/SAMJ.2019.v109i2.00011

Cerebrovascular accidents/strokes are a public health issue, which is common and serious worldwide. Globally, strokes have high mortality and morbidity rates. In South Africa (SA), stroke is a leading cause of morbidity. ${ }^{[1]}$ In recent years, advances in medical management of acute strokes have resulted in a decrease in the global mortality rate of strokes, but despite this, many survivors remain with significant impairments. ${ }^{[2]}$ According to Duncan et al. ${ }^{\left[{ }^{[3]}\right.} 40 \%$ of patients who survive a stroke are left with moderate disabilities and 15 - $30 \%$ with severe disabilities. It can be assumed that the greatest health effect and financial burden on stroke survivors and their families are the possible long-term disabling effects. ${ }^{[4]}$

Rehabilitation initiated early after a stroke has been shown to assist with reducing complications and residual post-stroke functional disabilities. A reduction in functional disability, as well as a reduced incidence of complications, results in a greater quality of life for stroke survivors and a decrease in potentially expensive long-term care costs. ${ }^{[2,3]}$ However, despite the increasing evidence that poststroke rehabilitation is beneficial, many healthcare professionals are unaware of stroke survivors' potential for improvement and reduction of secondary complications. ${ }^{[5]}$

Although there is growing evidence that post-stroke rehabilitation is beneficial, ${ }^{[6]}$ there is limited research into the specificities of post-stroke rehabilitation interventions. Therefore, information on the complexities and specific fundamentals of post-stroke rehabilitation tends to rely mainly on expertise of individual healthcare professionals. ${ }^{[4]}$

\section{Clinical effects of a stroke}

The disabling effects of a stroke are dependent on the affected areas of the brain. By far the most common result of stroke is motor impairment, affecting $~ 80 \%$ of patients; it involves weakness of the face, arm and leg (in varying degrees) on one side of the body. ${ }^{[2]}$

The brain can be divided into four main areas: the left/dominant hemisphere, the right/non-dominant hemisphere, the cerebellum and the brainstem (both the cerebellum and brainstem fall within the area known as the posterior fossa). These divisions make it easier to list and discuss the possible effects of stroke. The Oxfordshire stroke classification simplifies syndromes into either haemorrhage or infarct and localises clinical presentations according to the area of damage.
The broad divisions are: total anterior circulation stroke (TACS), partial anterior circulation stroke (PACS), lacunar stroke (LACS) and posterior circulation stroke (POCS). ${ }^{[7]}$ These clinical stroke syndromes have a mix of neurological presentations (hemiparesis, sensory changes, visuospatial problems and cognitive impairments), and functional outcomes can be predicted according to the various syndromes described.

\section{What is rehabilitation?}

Post-stroke rehabilitation is a patient-centred, goal-driven process that attempts to maximise the functional independence of patients who suffer from a variety of stroke-related disabilities. ${ }^{[2]}$ The main aim of post-stroke rehabilitation is to assist the stroke survivor to return to their premorbid functioning (or as close to it as possible) within their family environment, community environment and where possible, work environment. Rehabilitation can be delivered in an inpatient or outpatient setting. In an SA healthcare context, inpatient rehabilitation is preferable, as outpatient rehabilitation tends to lack the intensity offered by the former. Although admission criteria may differ from one rehabilitation centre to the other, typically a stroke patient qualifies for inpatient rehabilitation if they require $\geq 3$ modalities of interventions or if they are unable to transfer independently. ${ }^{[1]}$ According to Bryer et al. ${ }^{[1]}$ most SA stroke patients are treated in the public healthcare sector. Because of a lack of inpatient stroke rehabilitation resources in this sector, efforts have been made to improve the availability and quality of home-based care and rehabilitation, as well as community-based rehabilitation services.

\section{Focus areas for post-stroke rehabilitation}

Post-stroke rehabilitation is delivered most effectively and efficiently by an interdisciplinary team comprised of healthcare professionals working together to achieve common, shared goals for a patient's rehabilitation. Constant and effective communication between team members and the patient allows for a more holistic approach. Good communication among team members also allows individual professionals to use their expertise to build on that of another team member. ${ }^{[8]}$ An interdisciplinary team usually includes nurses, medical 
practitioners, physiotherapists, occupational therapists, speech and language therapists (all of whom are experienced in rehabilitation), a social worker and a dietitian. In addition to these disciplines, other professionals are included in the rehabilitation team, depending on individual patients' needs and wants, e.g. a psychologist or trauma counsellor.

There are specific areas of focus for an interdisciplinary team involved in post-stroke rehabilitation, which should commence in the acute setting or intensive care unit (ICU) and be continued in the formal rehabilitation setting. The five main areas of focus are given below.

\section{Reducing risk factors and preventing complications}

A high priority of stroke rehabilitation is reducing stroke risk factors (e.g. by treating and managing comorbidities such as hypertension). Suffering a stroke places a patient at high risk of a recurrence; therefore, careful management of risk factors is essential. ${ }^{[9]}$ Another important priority is the prevention and treatment of secondary complications commonly associated with strokes. Secondary complications can adversely impact on a patient's rehabilitation. These include:

- Spasticity. A patient's mobility can be limited greatly by spasticity. A number of therapeutic interventions may help: passive stretching, splinting and posture and movement therapy. ${ }^{[3]}$ Oral agents such as baclofen and clonazepam and parenteral therapies such as botulinum toxin injected into affected muscle are helpful. ${ }^{[1]}$ Poorly managed spasticity not only reduces functional independence, but results in the potential for painful contractures, making hygiene, especially in the contracted hand, a challenge.

- Pain (central and nociceptive). Nociceptive pain in the shoulder is common in stroke patients. Physiotherapists are often able to assist with strapping, massage or passive movements. Pharmacological management might be required. Central or neuropathic pain is another common consequence of stroke. The use of the gabapentinoids, such as pregabalin and gabapentin, is effective. However, these medications can have cognitive side-effects.

- Pressure ulcers. The risk of developing pressure ulcers is high in stroke patients, attributed mainly to their decreased mobility. Regular turning when in bed, adequate nutrition and pressure care education for the patient and their families/caregivers are helpful preventive strategies. ${ }^{[1]}$ Should a pressure ulcer develop, early assessment and treatment by a physician and nurses experienced in pressure ulcer management are needed.

- Deep vein thrombosis and pulmonary embolism. A stroke patient's decreased mobility puts them at high risk for deep vein thrombosis and pulmonary embolism. Early prophylactic management includes the use of low-molecular-weight heparin and compression stockings. Physicians and nurses need to be alert to any signs and symptoms related to deep vein thrombosis or pulmonary embolism to ensure timely diagnosis and correct treatment.

- Urinary and faecal incontinence. Incontinence is a common complication of stroke. The use of an indwelling catheter in the acute management of a stroke patient is helpful with fluid management, preventing urinary retention and preventing skin breakdown. However, the prolonged use puts a patient at risk for urinary infections. These often require antibiotic intervention, with multiresistant organisms frequently found. Infections result in a patient not feeling well enough to participate in their rehabilitation. Faecal incontinence is a less common long-term problem, as it often resolves within the first 2 weeks after a stroke. Faecal impaction syndromes, however, are common and preventive management is essential.
- Aspiration pneumonia. Patients with dysphagia are at risk of aspiration. Interventions by a speech and language therapist help to manage this risk. However, a physician should have a low threshold for the early treatment of any signs and symptoms of aspiration before the patient develops aspiration pneumonia. When in doubt, patients should be fed by nasogastric tube until fully cleared for safe oral intake by a speech and language therapist.

\section{Therapy-specific rehabilitation}

The therapeutic disciplines traditionally involved in post-stroke rehabilitation are physiotherapy, occupational therapy and speech and language therapy. The roles of a physiotherapist and an occupational therapist often seem to overlap. ${ }^{[10]}$ Although both disciplines work predominantly with the motor impairments of stroke patients, their approach to and focus on the impairments differ. Physiotherapists focus on the correct positioning of hemiplegic patients as well as early mobilisation and mobility of patients, whereas an occupational therapist works towards the resumption of activities of daily living (e.g. grooming and dressing), often incorporating the use of assistive devices. The occupational therapist is also involved in early cognitive screening, and cognitive rehabilitation is undertaken in conjunction with the speech and language therapist. ${ }^{[1,11]}$ Return-towork assessment and driving assessment are normally the domain of the occupational therapist. Many stroke patients suffer from a range of cognitive, communicative and swallowing impairments. Speech and language therapists are highly trained to manage and treat such impairments. ${ }^{[11]}$

\section{Patient and family/caregiver education and involvement} It is very important that patients and their families/caregivers are seen as part of the interdisciplinary team from the start. They must receive education on the causes and consequences of and recovery from strokes. ${ }^{[3]}$ There should be constant interaction between the interdisciplinary team and the patient and their families/caregivers regarding the rehabilitation goals and progress.

\section{Psychological/psychiatric support}

There are a variety of neuropsychiatric sequelae after stroke - the most common being depressive symptoms. Post-stroke depression develops in $25-75 \%$ of patients. ${ }^{[3]}$ However, it is not unusual for stroke patients to experience periods of emotionalism. Feelings of fear, anxiety, a sense of grief for their physical losses/changes, as well as other symptoms similar to those seen in depression might manifest. These normally dissipate with time and can be adequately managed with the support of the interdisciplinary team, including counsellors. It is therefore important to include a psychologist or counsellor on the team. If the abovementioned symptoms persist and interfere with a patient's other therapies, given the commonality of post-stroke depression, the rehabilitation physician should have a high index of suspicion for making the diagnosis. Subsequent pharmacotherapeutic management with selective serotonin reuptake inhibitors or heterocyclics is usually warranted and referral to a psychiatrist might be needed.

\section{Nutrition and diet}

Nutrition is a very important post-stroke rehabilitation intervention. A dietitian should be part of a stroke patient's interdisciplinary team from the start of the rehabilitation process. Malnourishment is a common problem, either because of swallowing difficulties or because of problems communicating, which may result in patients being unable to adequately express their dietary requirements. The rehabilitation process is a very demanding one and a malnourished 
patient does not have the energy or the mental focus to fully participate in all aspects of their rehabilitation.

\section{Discharge and outcomes}

Discharge planning is a very important part of post-stroke rehabilitation. Provisional discharge plans made in the early stages of rehabilitation (which can then be altered if a patient's needs change during their rehabilitation process) allow family or caregivers ample time to put systems in place (e.g. make any necessary alterations to their homes or organise outside carer employment and environments). If there is a possibility of returning to work, this should also be included in the discharge planning. Early and ongoing communication between the interdisciplinary team and the patient's employers can greatly assist with the reintegration into the workspace. The support given to the patient by their family and caregivers after discharge from inpatient rehabilitation plays a significant role in determining a patient's long-term outcome.

Another relatively reliable indicator of stroke prognosis is the degree of motor impairment. There are also other impairments (such as dysphagia and urinary incontinence) regarding severe disability levels after stroke. However, the heterogeneity and multilayered nature of strokes make it difficult to accurately predict post-stroke prognosis. A patient's post-stroke rehabilitation must not discontinue on discharge from an inpatient rehabilitation unit.

Continued rehabilitation for up to a year after stroke has been shown to assist in the overall long-term outcome of a patient. ${ }^{[1]}$ Referrals to appropriate healthcare professionals need to be made timeously by the inpatient interdisciplinary team to ensure that there is a good structure in place for continuation of a patient's rehabilitation after discharge.
Declaration. None.

Acknowledgements. None.

Author contributions. Both authors were involved in the literature search and writing of this review.

Funding. None.

Conflicts of interest. None.

1. Bryer A, Connor M, Haug P, et al. South African guideline for the management of ischaemic stroke and transient ischaemic attack 2010: A guideline from the South African Stroke Society (SASS) and the SASS Writing Group. S Afr Med J 2010;100(11):750-776. https://doi.org/10.7196/samj.4422

2. Brewer L, Horgan F, Hickey A, Williams D. Stroke rehabilitation: Recent advances and future therapies. Brewer L, Horgan F, Hickey A, Williams D. Stroke rehabilitation:
QJM 2013;106(1):11-25. https://doi.org/10.1093/qimed/hcs174

3. Duncan P, Zorowitz R, Bates B, et al. Management of adult stroke rehabilitation care: A clinical practice guideline. Stroke 2005;36(9):100-143. https://10.1161/01.STR.0000180861.54180.FF

4. Langhorne P, Bernhardt J, Kwakkel G. Stroke rehabilitation. Lancet 2011;377(9778):1693-1702. https:// doi.org/10.1016/s0140-6736(11)60325-5

5. Xorowitz R. Stroke rehabilitation: An overview of existing guidelines and standards of care. US Neurol 2011;7(1):41-45. https://doi.org/10.17925/USN.2011.07.01.41

6. Duncan P, Horner RD, Reker DM, et al. Adherence to postacute rehabilitation is associated with functional recovery in strokes. Stroke 2002;33(1):167-178. https://doi.org/10.1161/hs0102.101014

7. Amarenco P, Bogousslavsky J, Caplan LR, et al. Classification of stroke subtypes. Cerebrovasc Dis 2009;27(5):493-501. https://doi.org/10.1159/000210432

8. Jessup R. Interdisciplinary versus multidisciplinary care teams: Do we understand the difference? Austr Health Rev 2007;31(3):1-2. https://doi.org/10.1071/ah070330

9. Bates B, Choi JY, Duncan P, et al. Veteran Affairs/Department of Defence clinical practice guideline for the management of adult stroke rehabilitation care: Executive summary. Stroke 2005;36(9):2049-2056. https://doi.org/10.1161/01.STR.0000180432.73724.AD

10. Booth J, Hewison A. Role overlap between occupational therapy and physiotherapy during inpatient stroke rehabilitation: An exploratory study. Abstract, Qualitative Based Practice Conference. Coventry University, UK, 14 - 16 May 2001. I Interprof Care 2002;16(1):31-40. https://doi. Coventry University, UK, $14-$

11. Dragga A. The role of speech-language pathologists in stroke rehabilitation. Rhode Island Med J 2015;98(12):20-22.

Accepted 27 December 2018. 